\title{
EDITORIAL
}

\section{Advancements in Molecular Medicine and Tailor-Made Therapeutics}

\author{
Rehan Imad, Talat Mirza \\ Department of Research, Ziauddin University, Karachi, Pakistan. \\ https://doi.org/10.36283/PJMD10-2/001
}

The marvelous success in the field of molecular therapeutics is being witnessed by science over the past few decades. From single cell RNA sequencing to circulating cell free DNA detection in the blood, astonishing accomplishments have been achieved. These advancements, however, are still confronting the challenges of the old times, such as non-specificity, inter-individual variability, and off-target effects. Molecular medicine as a science has provided a platform to address these problems, by tailoring according to the molecular and genetic profile of the individuals to increase the specificity of the treatment, to reduce variability, and to minimize adverse side effects. Advancements in this field are opening new vistas for shifting the paradigm across therapeutics and drug discovery, made possible through better understanding of the disease process at molecular level'.

Recent molecular profiling by Next Generation Sequencing (NGS) is helping researchers to identify molecular targets which can be utilized for the management of challenging issues such as metastatic carcinomas. This information has also been used for precision medicine in individual patients to adjust therapeutic regimen according to their need. In a post genomic era where sequence information of most of the organisms is readily available, approaches are now needed to be channeled to understand novel gene functions for their potential fundamental role in etiology of diseases ${ }^{2}$.

Multi-omics approach, in contrast to single omics investigations, deliver greater understanding of the disease process by means of transcriptomics, proteomics, metabolomics, genomics and microbiomics which are integrated through systems and computational biology, and bioinformatics ${ }^{3}$.

Gene therapy has provided the concept of treating disease at molecular level. The first approved gene therapy was performed in early 90s for adenosine deaminase (ADA) deficiency which causes severe combined immunodeficiency (SCID) by inserting functional ADA into patient's T cells ${ }^{4}$. Further advancements with time have improved precision and efficiency of gene therapy leading to the advent of more sophisticated tools such as TALEN and CRISPR/Cas9 ${ }^{5}$. What makes CRISPR/Cas9-based gene editing so exciting is the high target specificity conferred by guide RNA. More recently, CRISPR system has also been employed for the diagnosis of covid-19 as a possible alternate to the conventional diagnostic methods ${ }^{6}$.

Genome-wide association studies (GWAS) and microarray-based analysis integrates new information generated in the lab with the clinical settings, and medical practitioners need to take maximum benefit from the factual evidences provided by the researchers. This can help physicians to find the disease prognosis of patients with the same symptoms and hold the potential for powerful classification of patients with prediction of treatment outcome.

The use of inhibitory RNAs, synthetic compounds structurally designed using computational biology and bioinformatics, nucleic acid-based loop mediated isothermal amplification (LAMP) and drug repositioning tools can be effectively used to deliver ground breaking technological advancements pertaining to the field of molecular medicine. LAMP is a Food and Drug Administration (FDA) approved diagnostic assay, which has been given emergency use authorization for diagnosis of covid-19 infections in setups where QPCR is not available.

A new era of molecular medicine sciences based on technical revolutions has already been ushered in Pakistan as well. In-depth information of genetic and molecular mechanism of diseases has given us a vast insight into the broader spectrum of prognostic, diagnostic, therapeutic and preventive strategies. It is high time when our medical community should work with the scientific community in close liaison to discover new knowledge and to identify improved therapies for betterment of humanity at large, leading to fruitful physician-scientist relationship. 


\section{REFERENCES}

1. Schenone M, Dančík V, Wagner BK, Clemons PA. Target identification and mechanism of action in chemical biology and drug discovery. Nat Chem Biol. 2013;9(4): 232-240.

2. Austin ED, West J, Loyd JE, Hemnes AR. Molecular medicine of pulmonary arterial hypertension: from population genetics to precision medicine and gene editing. Am J Respir Crit Care Med. 2017;195(1):23-23. 3. Nalbantoglu S, Karadag A. Introductory Chapter: Insight into the OMICS Technologies and Molecular Medicine. InMolecular Medicine 2019. IntechOpen [cited 2019 Oct 13]. Available from: https://www.intechopen.com/books/molecular-medicine/introductory-chapter-insight-into-the-omics-tec hnologies-and-molecular-medicine

4. Blaese RM, Culver KW, Miller AD, Carter CS, Fleisher T, Clerici M, et al. T lymphocyte-directed gene therapy for ADA-SCID: initial trial results after 4 years. Sci. 1995;270(5235):475-480.

5. Goodwin M, Lee E, Lakshmanan U, Shipp S, Froessl L, Barzaghi F, et al. CRISPR-based gene editing enables FOXP3 gene repair in IPEX patient cells. Sci Adv. 2020;6(19):1-16.

6. Broughton JP, Deng X, YU G, Fasching CL, Servellita V, Singh J, et al. CRISPR-Cas12-based detection of SARS-CoV-2. Nat Biotechnol. 2020; 38(7):870-874. 